\title{
ANATOMY OF THE FORELIMB IN THEROPITHECUS OSWALDI
}

WHITEHEAD, Paul F., Div. of Vertebrate Zoology, Peabody Museum of Natural History, Yale University 06511

A sample of over 170 hand, wrist, forearm and upper arm bones, from Olorgesailie DE/89 Horizon B and from Olduvai Gorge, was examined in this study. Elements include a trapezoid and centrale, capitates, hamates, scaphoids, lunates, pisiforms, triquetra and second, third, fourth and fifth metacarpals, ulnae, radii, humerii and scapulae. The fossils are different in several functionally significant ways from modern papionids. The angulation between the dorsal and palmar halves of the scaphoid is less acute. The helicoidal facet of the hamate, for articulation with the triquetrum, is radio-ulnarly shorter than in modern cercopithcoids; this would limit ulnar deviation. The hamulus is disto-proximally shorter, relative to the hamate body, than in digitigrade cercopithecoids, knuckle-walkers or fist-walkers. The distal hamate surface has the division into distinct, separate fourth and fifth metacarpal facets that is present in palmigrade cercopithecoids. Intermetacarpal facets are generally bissected by deep grooves, indicating strong intermetacarpal ligaments. The facet on the capitate for articulation with the second metacarpal, and the corresponding facet on the metacarpal, indicate that there was a functional division between the second and third metacarpals. The two extremes of retroflexion of the medial epicondyle of the humerus are present in the sample of humerii: some specimens closely resemble the angulation found in arboreal colobus quereza and cercopithecus albogularis, while others are similar to terrestrial papio and Erythrocebus. The extremes of bifurcation and angulation of the radial notch are present in the sample of ulnae. Some specimens have the arboreal condition of a single, basically lateral-facing facet; others have the terrestrial split radial notch. The terrestrial condition is found where movement is confined to the parasaggital plane, with the arm in full pronation. The more lateral orientation of the radial notch appears to indicate more capacity for pronation/supination. The retroflexed olecranon is indicative of terrestriality; all of the examined ulnae have the short, grooved, angled olecranon typical of terrestrial monkeys. The associated specimens from MCK II illustrate that the features can appear as a mosaic: "tarrestrial" olecranon, retroflexed medial epicondyle but "arboreal" radial notch. The brachialis mark is more anteriorly-oriented than in modern cercopithecoids. 